\title{
On Optimal Resampling of View and Illumination Dependent Textures
}

\author{
Jiří Filip* \\ Michael J. Chantler \\ Michal Haindl \\ Heriot-Watt University \\ Heriot-Watt University \\ ÚTIA, AS ČR
}

\begin{abstract}
The use of illumination and view dependent textural information is one way to capture the realistic appearance of genuine materials. One example of such data is the bidirectional texture function. The main disadvantage of these data, that makes their further application very difficult, is their massive size. Perceptually-based methods can determine optimal uniform resampling of these data that allows considerable reduction of a number of view and illumination dependent samples. In this paper we propose to achieve this goal by means of a psychophysical study, comparing original data rendering with rendering of their uniformly resampled version over the hemisphere of illumination and view dependent textural measurements. The resampling was done separately for elevation and azimuthal angles as well as in illumination and view space. Our results shown promising consequences for compression and modeling algorithms using this kind of massive data.
\end{abstract}

CR Categories: I.3.7 [Three-Dimensional Graphics and Realism]: Color, shading, shadowing, and texture- [I.4.7]: Image Processing and Computer Vision-Feature Measurement-Texture J.4 [Social and Behavioral Sciences]: Psychology

Keywords: BTF, optimal resampling, visual degradation, phychophysical experiment, texture compression

\section{Introduction}

In many industrial sectors, demand is currently increasing for accurate virtual representation of real-world materials. Important application areas include safety simulations and computer-aided design. In the first area, the main concern is choosing the right material to fulfill given safety limits of reflectance, while in the second the aim is to avoid costly and time consuming design cycles of material selection, solid model production and visual evaluation. These tasks, among others, require accurate photo-realistic representations of real material samples dependent on different illumination and viewing conditions.

One such representation is the Bidirectional Reflectance Distribution Function [Nicodemus et al. 1977] (BRDF). The BRDF is defined as the ratio of radiance reflected from the material (outgoing) to the illuminating radiance (incoming) for all possible pairs of incoming $\omega_{i}\left(\theta_{i}, \phi_{i}\right)$ and outgoing $\omega_{v}\left(\theta_{v}, \phi_{v}\right)$ directions (see Fig. 1), resulting in a four-dimensional monospectral function $\operatorname{BRDF}\left(\theta_{i}, \phi_{i}, \theta_{v}, \phi_{v}\right)$. This function possesses two important properties; energy conservation and reciprocity of incoming and outgoing directions. As the BRDF captures the reflectance of only a small or averaged portion of the material surface, it is most applicable to surfaces without texture such as paints and similar finishes. Although spatially varying BRDFs can be used to describe textured

\footnotetext{
*e-mail: filipj@macs.hw.ac.uk
}

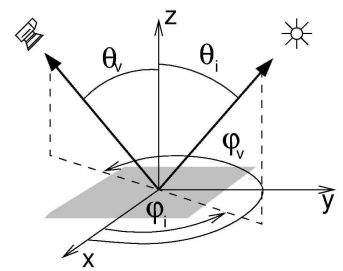

Figure 1: Relationship between illumination and viewing angles within texture coordinate space.

materials [Pellacini and Lawrence 2007], it is limited by its properties to smooth and opaque surfaces. The first real illumination/view dependent surface texture representation was the Bidrectional Texture Function (BTF), introduced in [Dana et al. 1999]. A BTF is a six-dimensional function representing the appearance of a sample surface for variable illumination and view directions. Compared to a four-dimensional BRDF, a BTF depends on two additional parameters, a planar position $(x, y)$ over a material surface, resulting in monospectral function $\operatorname{BTF}\left(x, y, \theta_{i}, \phi_{i}, \theta_{v}, \phi_{v}\right)$. The BTF represents such effects as masking, shadowing, inter-reflections and sub-surface scattering. During recent years, different BTF measurement systems have appeared, based on different principles each offering different advantages and disadvantages. Although material visualization using BTFs provide superb visual quality, even an average BTF sample (e.g., 256x256) often reaches gigabytes in size. This data size can be edited almost interactively by careful data management and empirical editing operators [Kautz et al. 2007]. However, it is still beyond the real-time rendering capabilities of current graphics hardware, and so there have been many recent research attempts to develop an efficient compression techniques that allow computationally cheap reconstruction and visualization of BTFs [Müller et al. 2005]. All such methods compress a full BTF sample, which often leads to extreme computational and excessive storage demands.

In this paper we aim to reduce the size of original BTF datasets, without loss of visual quality. To achieve this we propose a psychophysical study of various uniform BTF resampling schemes performed on eight datasets. This paper starts with recapitulation of published work in this research area in Section 2. Section 3 describes the experimental dataset and proposes several resampling schemes applied on these data. The evaluation of these schemes by psychophysical experiment is shown in Section 4. Discussion over the obtained results and their possible applications are in Section 5 while Section 6 concludes the paper and proposes directions for further development.

\section{Prior Work}

To the best of our knowledge, there are few publications on psychophysical analysis of view and illumination dependent texture data. Several papers investigate influences of light position, material reflectance, view position, or surface shape [Fleming et al. 2003], [Lawson et al. 2003], [te Pas and Pont 2005a], [te Pas and Pont 2005b], [Ramanarayanan et al. 2007]. [Pellacini et al. 2000] derived a psychophysically-based model of light reflection with two perceptually meaningful uniform dimensions. [Matusik et al. 2003] performed psychophysical tests showing consistent transitions in perceived properties between different BRDFs. Meseth et al [2006] shows a study comparing performance of material photographs, 


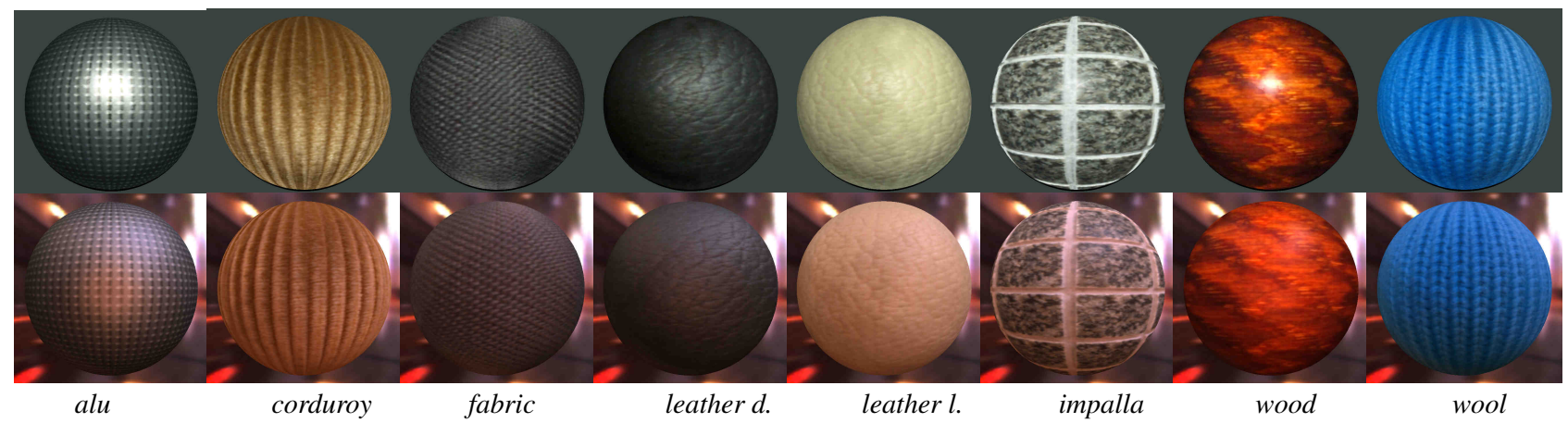

Figure 2: Examples of used BTF samples illuminated by point-light and environment illumination.

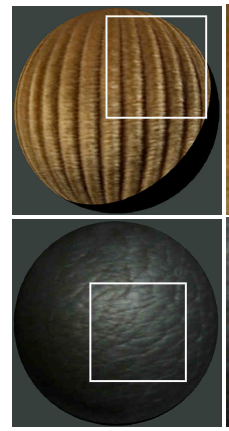

original data using images:

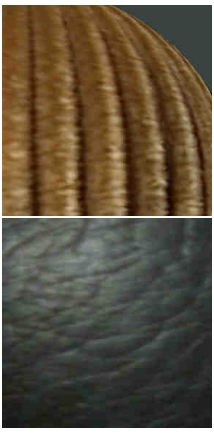

orig. $81 \times 81$

6561

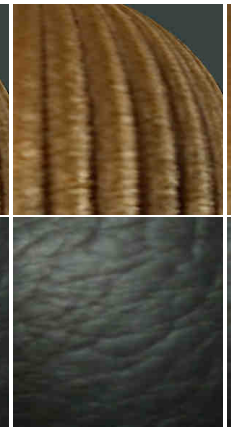

$\mathbf{A} \times \mathbf{A}$

1681

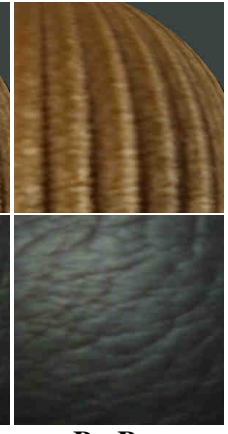

$\mathbf{B} \times \mathbf{B}$

1681

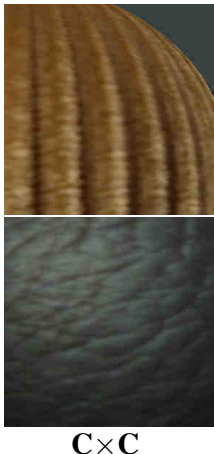

961
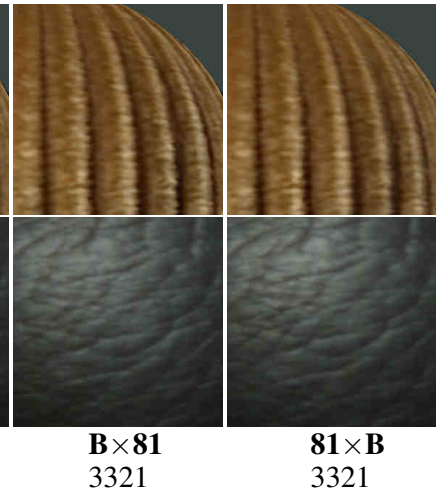

Figure 3: Examples of corduroy and leather d. BTF samples with view/illumination directions uniformly resampled in the proposed ways.

BTF rendering, and flat textures modulated by BRDFs for the same illumination condition. Methods mentioned above investigate influences of light, view, material reflectance, or shape but we are not aware of any other method dealing with optimal resampling of view/illumination dependent textural data.

\section{Proposed Data Resampling}

In this paper we have chosen BTF data as a typical example of simultaneous illumination and view dependent data. We have used the datasets from the Bonn BTF database ${ }^{1}$. For reduction of the size of processed datasets and simultaneously for enabling seamless covering of the test object, a BTF data tiling approach was applied. We have chosen BTF datasets corresponding to distinct types of real-world materials. Thus the following six different BTF datasets formed the subject of our experiment: aluminum profile (alu), corduroy fabric (corduroy), dark cushion fabric (fabric), artificial dark leather (leather $d$.), artificial light leather (leather l.), glazed tile with white pointing (impalla), lacquered wood (wood), and knitted wool (wool). Examples of these materials for both tested illumination environments are shown in Fig. 2. The original datasets have an angular resolution of illumination and viewing angles of $n_{i} \times n_{v}=81 \times 81$ (see Fig. 4-left).

In order to obtain considerable reduction of BTF dataset size we adopted three different BTF sampling schemes denoted as A, B, and $\mathbf{C}$ in Fig. 4. Each of the schemes is designed to fulfill uniform sampling in azimuthal angle $\phi$. While scheme A preserves original sampling of elevation angle $\theta$ but reduces the number of azimuthal samples along angle $\phi$, schemes $\mathbf{B}$ and $\mathbf{C}$ reduce sampling for both angles. While schemes $\mathbf{A}$ and $\mathbf{B}$ produce the same number of samples, i.e., 41 , scheme $\mathbf{C}$ reduces the number of samples even more aggressively yielding only 31 samples. Numbers

\footnotetext{
$1_{\text {http: } / / \text { btf.cs.uni-bonn. de/ }}$
}

of samples for individual levels of elevation angle $\theta$ for individual resampling schemes are given at the bottom of Fig. 4. Note that the view and illumination dependent data, i.e., BTF, require directional sampling of both illumination $\omega_{i}\left(\theta_{v}, \phi_{v}\right)$ and view directions $\omega_{v}\left(\theta_{v}, \phi_{v}\right)$. However, in these two directions we can adopt different sampling schemes without limiting practical usage of the data. Thus we decided to resample the original BTF datasets in five different test sets. The first three are straightforward and resample both $\omega_{i} \times \omega_{v}$ directions in the same way, using a combination of the same schemes $\mathbf{A} \times \mathbf{A}, \mathbf{B} \times \mathbf{B}$, and $\mathbf{C} \times \mathbf{C}$. The least two, used resample scheme $\mathbf{B}$ on either $\omega_{i}$ or on $\omega_{v}$. This resulted in resampling patterns of $B \times \mathbf{8 1}$ and $\mathbf{8 1} \times \mathbf{B}$. Consequently, the resampled datasets use the following numbers of BTF images:

$$
\begin{array}{lrrr}
\mathbf{A} \times \mathbf{A} & 1681 \text { images } & \mathbf{B} \times \mathbf{8 1} & 3321 \text { images } \\
\mathbf{B} \times \mathbf{B} & 1681 \text { images } & \mathbf{8 1} \times \mathbf{B} & 3321 \text { images } . \\
\mathbf{C} \times \mathbf{C} & 961 \text { images } & &
\end{array}
$$

Note that the original number of images in each dataset is 6561 . To avoid introduction of local errors into the original data by means of their down-sampling using local interpolation we used a two-step global interpolation scheme based on radial-basis functions [Carr et al. 2001]. In the first step the data for all illumination directions $\omega_{i}$ for fixed viewing direction $\omega_{v}$ are interpolated into a new illumination discretization scheme and these interpolated values for all combinations of $\theta_{i}$ and $\phi_{i}$ angles are further interpolated into a new viewing direction discretization scheme. Finally, all eight datasets were resampled in the five proposed ways (see Fig. 3) an together with the original datasets used in the following psychophysical experiment.

\section{Psychophysical Experiment}

The goal of the experiment was to analyze the influence of different illumination and view direction resampling schemes on the final appearance of rendered images. 


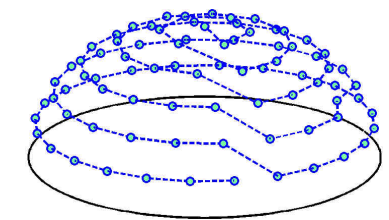

original BTF sampling 81 samples

$\theta=0^{\circ}: \# \phi=1$

$\theta=15^{\circ}: \# \phi=6$

$\theta=30^{\circ}: \# \phi=12$

$\theta=45^{\circ}: \# \phi=18$

$\theta=60^{\circ}: \# \phi=20$

$\theta=75^{\circ}: \# \phi=24$

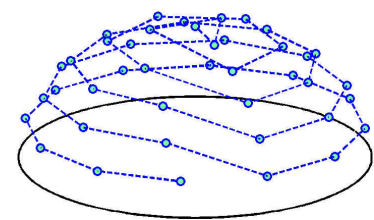

A: $\phi$ down-sampling 41 samples $\theta=0^{\circ}: \# \phi=1$

$\theta=15^{\circ}: \# \phi=3$

$\theta=30^{\circ}: \# \phi=6$

$\theta=45^{\circ}: \# \phi=9$

$\theta=60^{\circ}: \# \phi=10$

$\theta=75^{\circ}: \# \phi=12$

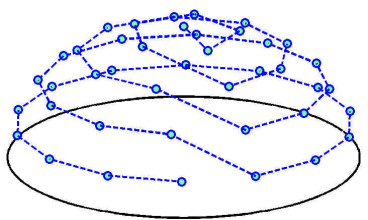

B: $\theta, \phi$ down-sampling 41 samples

$\theta=0^{\circ}: \# \phi=1$

$\theta=18.75^{\circ}: \# \phi=5$

$\theta=37.5^{\circ}: \# \phi=9$

$\theta=56.25^{\circ}: \# \phi=12$

$\theta=75^{\circ}: \# \phi=14$

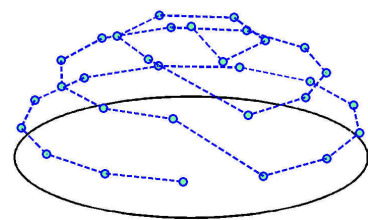

C: higher $\theta, \phi$ down-sampling 31 samples

$\theta=0^{\circ}: \# \phi=1$

$\theta=25^{\circ}: \# \phi=6$

$\theta=50^{\circ}: \# \phi=11$

$\theta=75^{\circ}: \# \phi=13$

Figure 4: Sampling of original BTF measurements (left) compared with its three tested resampled schemes: $\boldsymbol{A}-$ along azimuth $\theta, \boldsymbol{B}, \boldsymbol{C}-$ along azimuth $\theta$ and elevation $\phi$ angles. At the bottom are numbers of azimuthal samples for each elevation level.

Experimental Stimuli. As experimental stimuli we have used pairs of static images of size $800 \times 800$, representing a material BTF rendered on a $3 \mathrm{D}$ object. Each pair consisted of a rendering using the full original dataset and one using one of the five resampled datasets. Pairs of images were displayed simultaneously, side-byside. A sphere was used as a test object rendered for point-light and grace $^{2}$ illumination environments. The point-light was positioned on the top-left from a viewing position consistent with the surrounding physical illumination. The environment maps were approximated by a set of 144 discrete point-lights [Havran et al. 2005]. The background of the point-light illuminated stimuli, and the remaining space on the screen, was set to dark gray. Given eight material BTFs, five different resampling schemes proposed in Section 3 and two different illumination types, the total number of stimuli was 80 .

Participants. Eleven paid observers (six males, five females) participated in the experiments. All were students or university employees working in different fields, were less than 35 years of age, and had normal or corrected to normal vision. All were naive with respect to the purpose and design of the experiment.

Experimental Procedure. The participants were shown the 80 stimuli in a random order and asked a yes-no question: 'Can you detect any differences in the material covering the two spheres?'. There was a pause of two seconds between stimuli presentations, and participants took on average less than 40 minutes to perform the whole experiment. All stimuli were presented on a calibrated 20.1" NEC2090UXi LCD display $(60 \mathrm{~Hz}$, resolution $1600 \times 1200$, color temperature $6500 \mathrm{~K}$, gamma 2.2 , luminance $120 \mathrm{~cd} / \mathrm{m}^{2}$ ). The experiment was performed under dim room lighting. Participants viewed the screen at a distance of $0.9 \mathrm{~m}$, so that each sphere in a pair subtended approximately $9^{\circ}$ of visual angle.

Experimental Results Analysis and Discussion. When participants reported a difference between the rendered images their response was assigned a value of 1 , and otherwise 0 . By averaging the responses of all participants, we obtained psychometric data for eight tested BTF samples, two different illumination schemes and the five proposed resampling schemes. The following section analyses and discusses the results of the experiment.

\section{Results and Discussion}

Results of the experiment for all five test sets in are shown in Fig. 5 and Fig. 6. All graphs in the figures show perceptual values of observed differences between the renderings of original and resampled BTF data. Fig. 5 shows average participants' responses for resampling schemes $\mathbf{A} \times \mathbf{A}, \mathbf{B} \times \mathbf{B}$, and $\mathbf{C} \times \mathbf{C}$. Fig. 6 illustrates responses for resampling schemes $\mathbf{8 1} \times \mathbf{B}, \mathbf{B} \times \mathbf{8 1}$, and $\mathbf{B} \times \mathbf{B}$. Both

\footnotetext{
$2_{\text {http: / / www . debevec.org }}$
}

figures show results for point-light (a) and grace environment illumination (b). The graphs include error-bars representing twice the standard error. Additionally, we performed Cochran Q-test [Cochran 1950] on the original dichotomous data obtained from the experiment. The obtained confidence intervals $\left(p_{Q}\right)$ corresponding to the tested datasets are shown below the individual graphs. Despite a relatively low number of tested subjects, we can see that for most of the datasets for point-light we have got quite significant values (the values fulfilling $75 \%$ significance test are underlined). The least significant values $\left(p_{Q}>0.2\right)$ were estimated for specular samples (alu and wood), where most of the resampling schemes blures specular highlights. The $p_{Q}$ values for grace environment are often less significant than for point-light, that is caused by less apparent differences between spheres in the stimuli.

In Fig. 5 we can observe a significant increase in perceived difference when resampling scheme $\mathbf{B} \times \mathbf{B}$ is used comparing to the scheme $\mathbf{A} \times \mathbf{A}$. This pattern was visible for both types of illumination. This means that the participants were much more sensitive to reduction of samples along elevation angle $\theta$ than to reduction of samples along azimuthal angle $\phi$. More aggressive down-sampling $\mathbf{C} \times \mathbf{C}$ did not introduce much more difference. Similar behavior can be found in Fig. 6, where resampling of view direction $\mathbf{8 1} \times \mathbf{B}$ introduces a significantly higher perceptual difference than resampling of illumination direction $\mathbf{B} \times \mathbf{8 1}$. When we compare resampling of view direction $\mathbf{8 1} \times \mathbf{B}$ with resampling of both direction $\mathbf{B} \times \mathbf{B}$ we cannot observe any particular increase in the perceptual difference. While the datasets of highly structured fabrics samples corduroy, fabric, and wool comply the most with the described behavior, the datasets corresponding to altogether smoother and more specular materials alu and wood have similar performance for point-light illumination regardless of the resampling scheme used. Fig. 5 suggests that using resampling based on scheme $\mathbf{A}$ can give even better visual performance, while using the same number of BTF images (3321 for $\mathbf{A} \times \mathbf{8 1}$ ). This allows considerable reduction of original 6561 BTF images, that are used as input data in many compression and modeling algorithms, without any particular perceptual error (see Fig. 3). This conclusion holds mainly for environment illumination which is, however, the prevailing type of illumination used in contemporary rendering systems.

\section{Conclusions}

The main goal of this paper was to determine optimal uniform resampling of view and illumination data without significant loss of their visual quality. This was achieved by means of psychophysical experiments using several resampling strategies applied on eight bidirectional texture function datasets corresponding to several natural and man-made materials. An analysis of the result- 
ing psychophysical data showed that optimal resampling of such data should reduce the number of samples along azimuthal and preserve original elevation view/illumination angles. Another important conclusion is that sampling of illumination direction can be significantly more sparse than sampling of view direction where the close-to-original sampling should be preserved to avoid significant blur in resampled data. Additionally, environment lighting is more convenient when resampled data are used, since the distortions introduced can be hidden by the convolution of the pixel with the underlying light pattern and is not so apparent to the observer. To sum up, our results have shown that even the uniform resampling of view/illumination data can often significantly reduce their size without significant perceptible difference. This simple result may benefit many compression, modeling, or rendering methods, which use this type of massive data.

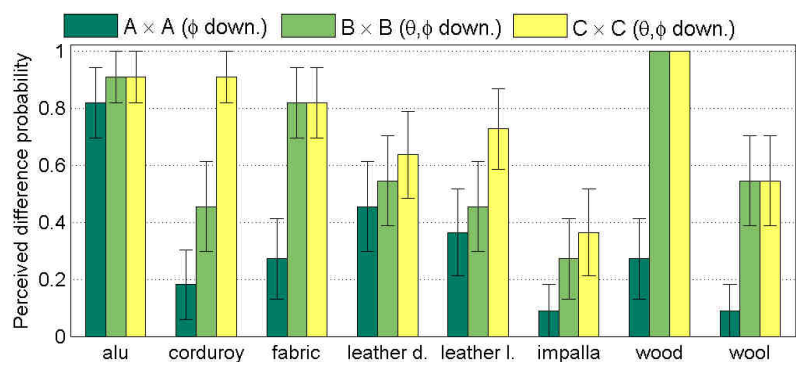

$\begin{array}{lllllllll}\text { (a) } p_{Q}: & 0.3679 & \underline{0.0046} & \underline{0.0421} & \underline{0.1738} & \underline{0.0907} & \underline{0.2231} & \underline{0.0025} & \underline{0.0017}\end{array}$

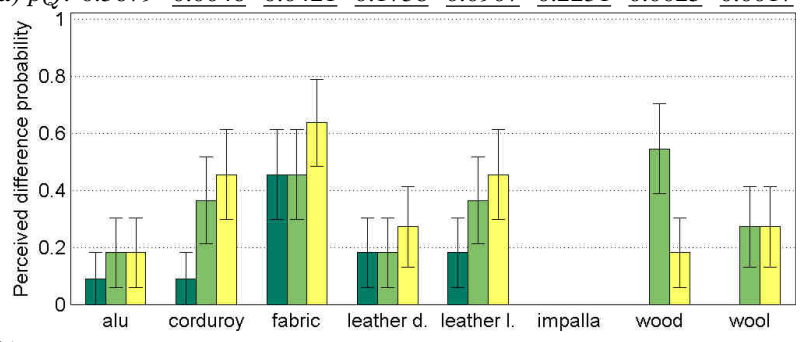

(b) $p_{Q}: \begin{array}{llllllll}0.7165 & \underline{0.0663} & 0.3679 & \underline{0.1054} & 0.3114 & 0.3679 & \underline{0.0342} & 0.3679\end{array}$

Figure 5: Results of the psychophysical experiment for all tested resampling schemes $\boldsymbol{A} \times \boldsymbol{A}, \boldsymbol{B} \times \boldsymbol{B}$, and $\boldsymbol{C} \times \boldsymbol{C}$ for different datasets and $(a)$ point-light and $(b)$ grace illumination environment.

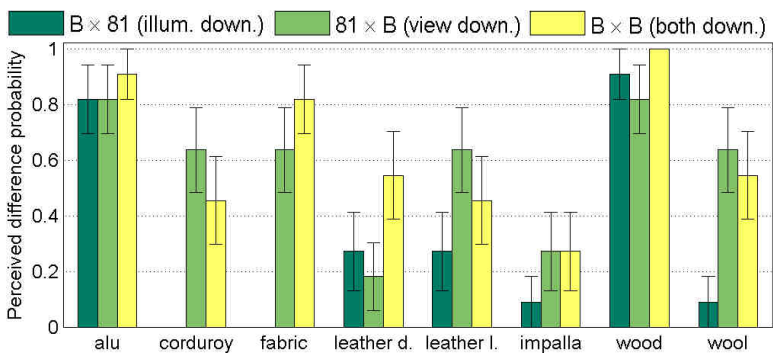

$\begin{array}{lllllllll}\text { (a) } p_{Q}: & 0.6065 & \underline{0.0013} & \underline{0.0004} & \underline{0.1561} & \underline{0.1561} & \underline{0.0970} & 0.3679 & \underline{0.0055}\end{array}$

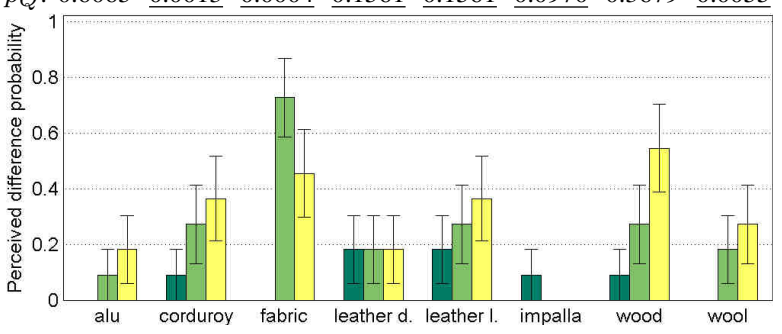

(b) $p_{Q}: \begin{array}{llllllll}0.7165 & 0.0388 & \underline{0.0055} & 1.000 & 0.8465 & 0.6065 & \underline{0.0281} & \underline{0.0663}\end{array}$

Figure 6: Results of the experiment for resampling of illumination/view/both respectively using the scheme $\boldsymbol{B}$ for different datasets and $(a)$ point-light and $(b)$ grace illumination environment.
Acknowledgments: This research was supported by the EC MarieCurie IEF No.41358, the EPSRC SMI grants GR/S12395/01 and EP/F02553X/1, and the GAČR grant 102/08/0593.

\section{References}

Carr, J., Beatson, R., Cherrie, J., Mitchell, T., Fright, W., McCallum, B., and Evans, T. 2001. Reconstruction and representation of 3D objects with radial basis functions. ACM SIGGRAPH 2001, 67-76.

COCHRAN, W. G. 1950. The comparison of percentages in matched samples. Biometrika, 37, 256-266.

DANA, K., VAN GiNnEKEN, B., NAYAR, S., AND KoENDERINK, J. 1999. Reflectance and texture of real-world surfaces. ACM Transactions on Graphics 18, 1, 1-34.

Fleming, R. W., Dror, R. O., AND Adelson, E. H. 2003. Real-world illumination and perception of surface reflectance properties. In Journal of Vision, vol. 3, 347-368.

Havran, V., Smyk, M., Krawczyk, G., Myszkowski, K., AND SEIDEL, H.-P. 2005. Interactive system for dynamic scene lighting using captured video environment maps. In Eurographics Symposium on Rendering, 31-42,311.

Kautz, J., Boulos, S., ANd Durand, F. 2007. Interactive editing and modelling of bidirectional texture functions. ACM Trans. Graph. 26, 3, 53.

Lawson, R., BÜlthoff, H., And Dumbell, S. 2003. Interactions between view changes and shape changes in picture picture matching. Perception 34, 12, 1465-1498.

Matusik, W., Pfister, H.P. BRAND, M., AND MCMillan, L. 2003. A data-driven reflectance model. ACM Transaction on Graphics 22, 3, 759-769.

Meseth, J., MÜller, G., Klein, R., RöDer, F., AND ARNOLD, M. 2006. Verification of rendering quality from measured BTFs. In Third Symposium on Applied Perception in Graphics and Visualization, 127-134.

Müller, G., Meseth, J., Sattler, M., Sarlette, R., AND KLEIN, R. 2005. Acquisition, synthesis and rendering of bidirectional texture functions. Computer Graphics Forum 24, 1 (March), 83-110.

Nicodemus, F., J.C., R., Hsia, J., Ginsburg, I., AND LIMPERIS, T. 1977. Geometrical considerations and nomenclature for reflectance. NBS Monograph 160, National Bureau of Standards, U.S. Dept. of Commerce, Washington, D. C., 1-52.

Pellacini, F., And Lawrence, J. 2007. Appwand: editing measured materials using appearance-driven optimization. ACM Transactions on Graphics 26, 3, 54:1-54:10.

Pellacini, F., Ferwerda, J., ANd Greenberg, D. 2000. Toward a psychophysically-based light reflection model for image synthesis. In 27th International Conference on computer Graphics and Interactive Techniques, 55-64.

Ramanarayanan, G., Ferwerda, J., Walter, B., AND BALA, K. 2007. Visual equivalence: towards a new standard for image fidelity. ACM Transactions on Graphics 26, 3, 76:176:10.

TE PAS, S., AND PONT, S. 2005. A comparison of material and illumination discrimination performance for real rough, real smooth and computer generated smooth spheres. In APGV'05, $57-58$.

TE PAS, S., AND PonT, S. 2005. Estimations of light-source direction depend critically on material BRDFs. Perception, ECVP Abstract Supplement 34, 212. 